\section{Fidelity and infidelity}

Miroslav Radman

T he apparent perfection of organisms and the accuracy of biological processes are still used in religious explanations of the origin of life. However, in real life it is survival, not fidelity, that is the ultimate virtue.

Because adaptability involves exploration of genetic possibilities to fit ecological niches, molecular infidelity and repetition are more likely to succeed than a precise, non-repetitive process. Only a tiny fraction of antibodies produced will ever be useful; the rest can be considered as mistakes. At least half of all human embryos fail during development. During chromosome segregation from a mother cell into two daughters, the polymerizing fibres (microtubules) do not know the exact location of the chromosomal target (the centromere) - they shoot and miss until one hits. A precise, single shot would often miss a target of uncertain position, whereas successive, imprecise firing will eventually lead to a hit. Selection at the level of molecules, cells and organisms may give the impression of designed perfection, but life's structures do not emerge by fully deterministic design.

What about the fidelity of enzymatic reactions? In the very precise process of DNA

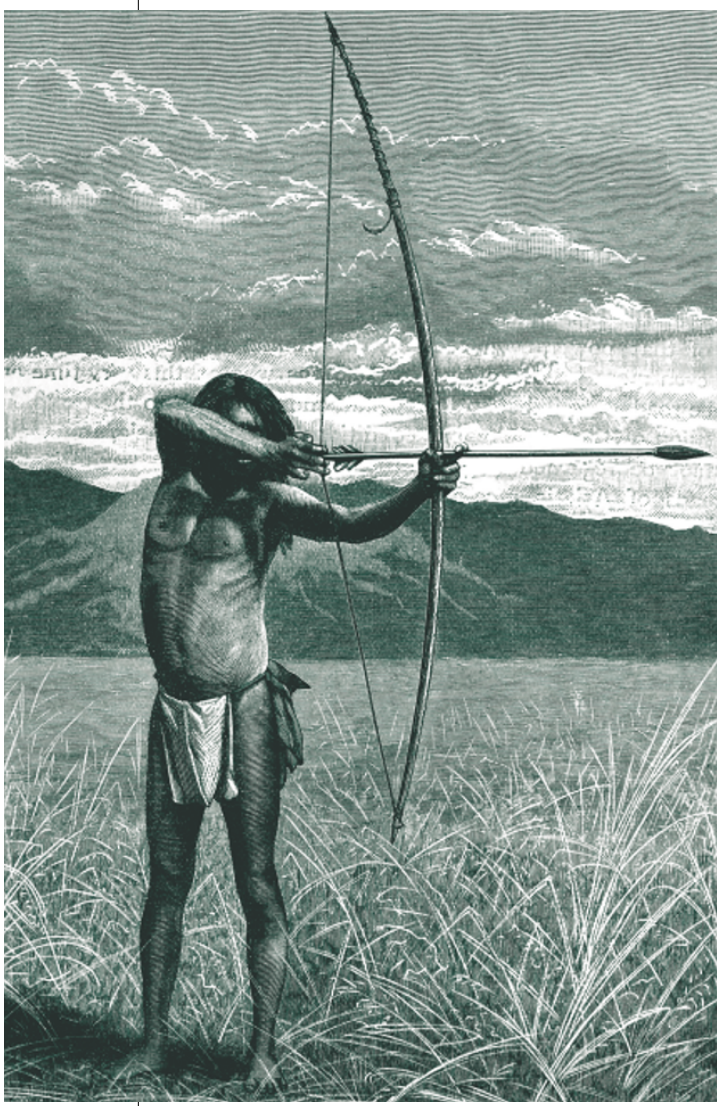

Moving target: roughly aimed shots lead to a hit. replication, accuracy is achieved by using a 'proofreading' system to remove erroneously inserted nucleotides, and then by qualitychecking the synthesized DNA using a mismatch-repair system that removes virtually all remaining mistakes. It would take too long to get it exactly right in the first place. DNA replication is efficient and therefore relatively imprecise, leaving mistakes to error-correction enzymes which are themselves efficient because their substrates are specific mistakes made by other enzymes.

John Hopfield and Jacques Ninio introduced the concept of 'kinetic' proofreading to explain the fidelity of molecular processes in which mistakes cannot be identified once they have been made. By delaying formation of the product, erroneous substrates are less likely to be transformed into it. Such mechanisms are seen in protein biosynthesis and may also be involved in presentation of antigens to T-cell receptors. In these cases, high fidelity is gained at the expense of efficiency.

Does nature attempt to achieve perfect fidelity using repair enzymes? Apparently not, because some bacterial mutants have increased fidelity of DNA or protein biosynthesis - an example is so-called StrR mutants, which are resistant to the antibiotic streptomycin. Streptomycin increases the error rate in protein biosynthesis; in its absence, StrR mutants have high fidelity, and grow more slowly than non-mutants. Because fidelity costs, it is optimized rather than maximized. Of course, error rates that are too high can cause death - bacteria and haploid yeast die from a genetic-error catastrophe when their mutation rate is increased 10,000-fold.

There is ample evidence of trade-offs between accuracy and efficacy. In protein synthesis, there are about 3 erroneous amino acids for every 10,000 correct ones; in transcription, about 1 nucleotide per 100,000 is wrong; and in DNA replication, about 1 nucleotide is wrong in every 10 billion. Proteins are functional molecules that are used up, oxidized, broken down and replenished by de novo synthesis. One-third of all proteins synthesized by normal human cells are immediately degraded by proteasomes because they have recognizable folding mistakes. Up to half of all messenger RNAs are erroneous and are therefore broken down. These clean-up systems probably contribute to the functional longevity of non-dividing cells such as neurons and cardiac muscle.

There is a huge investment in the fidelity of DNA replication, because genes are life's database. But when survival is threatened, even DNA fidelity is relaxed. For instance, when bacterial adaptation is limited by the available genetic diversity, genetically unstable

\section{Molecular evolution}

\author{
Errors and infidelity, even
}

wastefulness, can cause individual

failure, but they are also a source of innovation and robustness, ensuring the perpetuation of life.

populations adapt, at least in the short term, more rapidly than their stable counterparts.

Two classes of genes accelerate genetic variation by enhancing mutation and/or recombination in bacterial populations: stress-inducible wild-type genes and genes whose functional loss increases genetic variability; the former acts on individual cells and the latter on populations. Furthermore, genes encoding proteins under persistent selective pressure, such as a host's immunoglobulin genes or parasite target proteins/genes, show increased mutation rates. Thus, the fidelity of individual genes can be optimized.

There is also a down-to-earth reason for DNA infidelity: DNA replication itself! A topfidelity DNA-copying machine, such as the DNA-replication complex, can only copy chemically perfect DNA. But cellular DNA is not perfect, because of spontaneous oxidation, hydrolysis, alkylation, strand breaks and so on. As many as 300,000 such lesions may occur every day in every cell of a human body, most of which are dealt with by special repair systems. Each unrepaired lesion is a potentially lethal event that can stop the regular DNAreplication machinery. However, SOS, a class of unusual DNA polymerases, allows DNA replication to proceed despite the lesions.

Errors and infidelity, even wastefulness, can cause individual failure, but also provide innovation and robustness, ensuring the perpetuation of life. Nature does not exhaust itself for the sake of fidelity and perfectionism. Rather, errors are made, often repaired or discarded, but always tested as the source of blind innovation during the continuous adaptation to unpredictable environmental changes and challenges.

Miroslav Radman is at the Laboratory of Evolutionary and Medical Molecular Genetics, INSERM E9916,

and in the Department of Cell Biology, Université de Paris-V, 75730 Paris Cedex 15, France.

\section{FURTHER READING}

Caporale, L. H. (ed.) Molecular Strategies in Biological Evolution (New York Acad. Sci., 1999).

Keller, E. F. The Century of the Gene (Harvard Univ.

Press, Cambridge, Massachusetts, 2000).

Freidberg, E. C. Correcting the Blueprint of Life (Cold Spring Harbor Lab. Press, Cold Spring Harbor, New York, 1997). 